\title{
Hierarchical Connectivity and Connection-Specific Dynamics in the Corticospinal-Corticostriatal Microcircuit in Mouse Motor Cortex
}

\author{
Taro Kiritani, ${ }^{1}$ Ian R. Wickersham, ${ }^{2}$ H. Sebastian Seung, ${ }^{2}$ and Gordon M. G. Shepherd ${ }^{1}$ \\ ${ }^{1}$ Department of Physiology, Feinberg School of Medicine, Northwestern University, Chicago, Illinois 60611, and ${ }^{2}$ Howard Hughes Medical Institute and \\ Department of Brain and Cognitive Sciences, Massachusetts Institute of Technology, Cambridge, Massachusetts 02139
}

\begin{abstract}
The generation of purposive movement by mammals involves coordinated activity in the corticospinal and corticostriatal systems, which are involved in different aspects of motor control. In the motor cortex, corticospinal and corticostriatal neurons are closely intermingled, raising the question of whether and how information flows intracortically within and across these two channels. To explore this, we developed an optogenetic technique based on retrograde transfection of neurons with deletion-mutant rabies virus encoding channelrhodopsin-2, and used this in conjunction with retrograde anatomical labeling to stimulate and record from identified projection neurons in mouse motor cortex. We also used paired recordings to measure unitary connections. Both corticospinal and callosally projecting corticostriatal neurons in layer 5B formed within-class (recurrent) connections, with higher connection probability among corticostriatal than among corticospinal neurons. In contrast, across-class connectivity was extraordinarily asymmetric, essentially unidirectional from corticostriatal to corticospinal. Corticostriatal neurons in layer $5 \mathrm{~A}$ and corticocortical neurons (callosal projection neurons similar to corticostriatal neurons) similarly received a paucity of corticospinal input. Connections involving presynaptic corticostriatal neurons had greater synaptic depression, and those involving postsynaptic corticospinal neurons had faster decaying EPSPs. Consequently, the three connections displayed a diversity of dynamic properties reflecting the different combinations of presynaptic and postsynaptic projection neurons. Collectively, these findings delineate a four-way specialized excitatory microcircuit formed by corticospinal and corticostriatal neurons. The "rectifying" corticostriatal-to-corticospinal connectivity implies a hierarchical organization and functional compartmentalization of corticospinal activity via unidirectional signaling from higher-order (corticostriatal) to lower-order (corticospinal) output neurons.
\end{abstract}

\section{Introduction}

Determining the synaptic organization of the microcircuits that drive corticospinal output is an essential step toward a mechanistic understanding of motor cortex function (Phillips and Porter, 1977; Matsumura et al., 1996; Amassian and Stewart, 2003; Smith and Fetz, 2009; Georgopoulos and Stefanis, 2010; Lemon, 2010). Corticospinal neurons were the first cortical cell class for which recurrent excitation was physiologically described (Phillips, 1959). Subsequent efforts to characterize the local cellular sources of excitatory input, in terms of the laminar locations and projection classes of presynaptic neurons, have been facilitated by the ability to label corticospinal neurons with fluorescent retrograde tracers (Tseng et al., 1991; Kaneko et al., 1994). For exam-

Received Sept. 18, 2011; revised Jan. 30, 2012; accepted Feb. 23, 2012.

Author contributions: T.K., I.R.W., and G.M.G.S. designed research; T.K. performed research; I.R.W. and H.S.S. contributed unpublished reagents/analytic tools; T.K. and G.M.G.S. analyzed data; T.K., I.R.W., H.S.S., and G.M.G.S. wrote the paper.

The authors declare no competing financial interests.

This work was supported by NIH Grant NS061963 (to G.S.) and the Whitehall Foundation (to G.S.). We thank Mac Hooks and Karel Svoboda for comments.

Correspondence should be addressed to Dr. Gordon M. G. Shepherd, Morton 5-660, 303 E. Chicago Avenue, Chicago, IL 60611. E-mail: g-shepherd@northwestern.edu.

DOI:10.1523/JNEUROSCI.4759-11.2012

Copyright $\odot 2012$ the authors $\quad 0270-6474 / 12 / 324992-10 \$ 15.00 / 0$ ple, such experiments have identified projections from layer $2 / 3$ pyramidal neurons as a source of interlaminar excitatory input to corticospinal neurons (Kaneko et al., 2000; Weiler et al., 2008; Anderson et al., 2010). Experiments in primates using rabies virus for trans-synaptic tracing also indicate an excitatory input pathway from upper layers (Rathelot and Strick, 2006). However, the connectivity of corticospinal neurons with other deeper-layer excitatory neurons remains unclear. Of particular interest are "crossed" (callosally projecting) corticostriatal neurons, because these mediate distinct aspects of motor control (Turner and DeLong, 2000; Reiner et al., 2010). A fundamental question is: what type of cross talk-if any-occurs between corticospinal and corticostriatal neurons?

We addressed this question by using optogenetic and electrophysiological methods to stimulate and record from identified corticospinal and corticostriatal neurons in brain slices of mouse motor cortex. This approach allowed us to characterize the matrix of connections formed among and between corticospinal and corticostriatal neurons. The results delineate an excitatory microcircuit comprising four functionally specialized pathways, including connection-specific dynamics and a wiring configuration notable for a paucity of corticospinal-tocorticostriatal connections. 


\section{Materials and Methods}

Animals. All animal procedures followed the animal welfare guidelines of Northwestern University, the Society for Neuroscience, and the National Institutes of Health. Mice (C57BL/6J, male and female) were bred inhouse or were received as litters from Jackson Laboratories.

Deletion-mutant rabies virus for retrograde labeling with channelrhodopsin-2. A deletion-mutant rabies virus (RV) carrying channelrhodopsin-2 (ChR2) fused to the fluorescent protein Venus (Nagai et al., 2002) was generated following published methods (Wickersham et al., 2010). As previously described, RV possesses key properties ideally suited for retrograde transfection, as follows: (1) the RV has a tropism for axons; (2) deletion of the glycoprotein gene renders the RV incapable of transsynaptic spread; (3) transgenes are expressed at high levels; and (4) deletion-mutant RV has low cytotoxicity (Wickersham et al., 2007). We prepared RV carrying a previously described ChR2 construct (pCAGGSChR2-Venus; Addgene Plasmid 15753) (Petreanu et al., 2007). A similar ChR2-carrying RV has recently been described (Osakada et al., 2011). Viral titer as determined by infection of 293T cells was $1.3 \times 10^{9}$ infectious units per milliliter.

Stereotaxic injections. Mice (age: postnatal day 18-21) were anesthetized and positioned in a stereotaxic frame. For spinal cord injections, the cervical spinal column was exposed by a dorsal approach and a laminectomy was performed at the $\mathrm{C} 1-\mathrm{C} 3$ level, exposing the dorsal cord. Spinal injections were targeted to the ventral horn $(\sim 0.5 \mathrm{~mm}$ lateral to midline, $\sim 1 \mathrm{~mm}$ deep). For striatal injections, a small craniotomy was opened over the somatosensory cortex, and injections were targeted to the dorsolateral striatum $(0.0 \mathrm{~mm}$ posterior, $2.0 \mathrm{~mm}$ lateral, and $2.5 \mathrm{~mm}$ ventral to bregma). Injections were made with an oil hydraulic manipulator (Narishige MO-10) and Drummond Wiretrol capillary pipettes. For RV injections, the volume (1.2 $\mu \mathrm{l}$ for spinal and $0.1 \mu \mathrm{l}$ for striatal injections) of solution containing the virus was slowly (over $\sim 1 \mathrm{~min}$ ) injected into the target site. For bead injections, either red or green fluorescent microspheres (RetroBeads, Lumafluor) were used following published methods (Anderson et al., 2010). In some experiments, cholera toxin subunit B, Alexa Fluor 555 conjugate ( $1 \mathrm{mg} / 1 \mathrm{ml}$; Invitrogen) was used instead of red fluorescent beads.

Brain slice electrophysiology. Coronal brain slices $(300 \mu \mathrm{m})$ containing motor cortex were prepared (Anderson et al., 2010) at postnatal days 22-28, 4-10 d after RV injection. Recordings were targeted to neurons labeled with fluorescent beads or, in optogenetic control experiments, to neurons expressing fluorescent protein. Whole-cell recordings were made with borosilicate patch electrodes $(3-6 \mathrm{M} \Omega$ ) filled with potassiumbased intracellular solution consisting of the following (in $\mathrm{mM}$ ): 128 $\mathrm{KCH}_{3} \mathrm{SO}_{3}, 4 \mathrm{MgCl}_{2}, 10$ HEPES, 10 phosphocreatine, 4 ATP, 0.4 GTP, 3 ascorbate, and in some cases also Alexa Fluor 488 hydrazide $(50 \mu \mathrm{M}$; Invitrogen) and biocytin (3 mg/ml), pH 7.25, 290-295 mOsm. Multiple simultaneous whole-cell recordings were made from identified corticospinal and/or corticostriatal neurons using a custom fluorescence microscope built around a quadruple recording rig (Scientifica) running the acquisition control software package Ephus (www.ephus.org) (Suter et al., 2010). Data were Bessel filtered at $4 \mathrm{kHz}$ and sampled at $10 \mathrm{kHz}$. Recordings were made at $31-33^{\circ} \mathrm{C}$, maintained with an in-line heating system (Warner). Slices were superfused with artificial CSF containing the following (in mM): $127 \mathrm{NaCl}, 2.5 \mathrm{KCl}, 25 \mathrm{D}$-glucose, $25 \mathrm{NaHCO}_{3}, 1.25$ $\mathrm{NaH}_{2} \mathrm{PO}_{4}, 2 \mathrm{CaCl}_{2}$ and $1 \mathrm{MgCl}_{2}$ in mM, oxygenated with $95 \% \mathrm{O}_{2} / 5 \%$ $\mathrm{CO}_{2}$. Results were not corrected for $\sim 10 \mathrm{mV}$ liquid junction potential.

$C h R 2$ photostimulation. In control experiments, to assess the efficacy of photostimulating the ChR2-expressing neurons, the spiking responses of transfected neurons were monitored (whole-cell recording, currentclamp mode) while brief (3-5 ms) photostimuli were delivered by gating the output of a blue LED (M470L2; driver: 1.2 A, LEDD1B; Thorlabs). This analysis demonstrated highly efficient and reliable photostimulation of the transfected neurons, with a spike probability at or close to 1 for brief flashes of blue light. For the connectivity experiments, pairs of untransfected and bead-positive neurons were recorded while presenting photostimuli (duration: $3-5 \mathrm{~ms}$; power: $1.5 \mathrm{~mW} / \mathrm{mm}^{2}$ ) to excite the ChR2-positive neurons. The pairs were recorded either simultaneously or sequentially; in the latter case, identical stimulus parameters were used for both neurons.

Paired recordings. We established simultaneous whole-cell recordings from two to four bead-positive projection neurons (corticospinal and/or corticostriatal, in various combinations) and sequentially tested their interconnectivity by evoking a train (four pulses, $20 \mathrm{~Hz}$, followed by a pulse $500 \mathrm{~ms}$ later) of action potentials in one neuron while monitoring responses in the others. At least 20 trials were collected $(5 \mathrm{~s}$ intertrial interval) to test each connection. For analysis of paired recording datasets, traces were averaged over multiple trials, and EPSPs in response to the first action potential (AP) in the train were detected based on standard waveform criteria ( $<20 \mathrm{~ms}$ peak latency relative to presynaptic AP peak, $<7 \mathrm{~ms} 20-80 \%$ rise time). In particular, we calculated the noise level during a baseline interval $(100 \mathrm{~ms})$, the peak amplitude during a poststimulus response interval $(0-25 \mathrm{~ms}$ relative to the command signal to the presynaptic neuron), and identified events with signal-to-noise $(\mathrm{s} / \mathrm{n})$ ratios $>5$.

\section{Results \\ Retrograde transfection of cortical projection neurons by RV-ChR2}

Retrograde anatomical labeling provides a means to identify projection neurons, but functional evaluation of microcircuit organization entails selective neuronal stimulation. We therefore developed a tool for retrograde optogenetic labeling of projection neurons. Deletion-mutant RVs enable high-intensity expression of transgenes in retrogradely labeled projection neurons-without trans-synaptic spread (Wickersham et al., 2007, 2010). We generated RV carrying channelrhodopsin-2 fused with a fluorescent protein (RV-ChR2). When RV-ChR2 was injected into the spinal cords of $\sim 3$-week-old mice, and brain slices containing motor cortex were cut several days later, fluorescently labeled corticospinal neurons (Fig. $1 A$ ) reliably fired action potentials in response to optical stimulation (Fig. $1 B$ ). Control experiments established that transfected neurons had normal resting membrane potentials and input resistances and that their photoexcitability was consistently high (Fig. $1 C-F$ ). In this type of optogenetic photostimulation paradigm, the ChR2-expressing axons (in this case from $\mathrm{RV}$-infected projection neurons) remain highly photoexcitable in brain slices even when severed (Petreanu et al., 2007; Anderson et al., 2010). These characterizations establish that retrograde transfection with RV-ChR2 enables selective and reliable photostimulation of synaptic output from projection neurons.

\section{RV-ChR2 assay of corticospinal connections to corticospinal and corticostriatal neurons in layer $5 \mathrm{~B}$}

First, to evaluate corticospinal outputs to corticospinal and corticostriatal neurons, we transfected corticospinal neurons with RV-ChR2 (Fig. 2A) and also injected retrograde tracers (fluorescent beads) into the contralateral spinal cord and striatum (Fig. $2 B$ ). In brain slices of motor cortex prepared several days later (Fig. 2C), this labeling paradigm resulted in the following readily distinguishable populations of projection neurons: (1) RV-transfected neurons expressing both a fluorescent protein (Venus) and ChR2, with or without beads as well; and (2) untransfected neurons that were anatomically labeled with either of two colors of fluorescent beads. The distinct labeling patterns of the two retrograde techniques allowed us to target postsynaptic recordings to Venus-negative, bead-positive projection neurons. Consistent with the results of the control experiments reported above (Fig. 1), showing that photocurrents were reliably present in transfected neurons and absent in untransfected neurons, in no case did we 


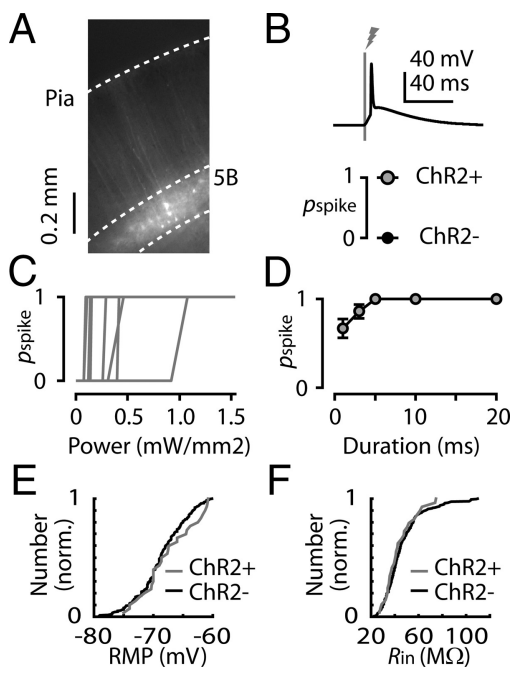

Figure 1. Photoexcitation of cortical projection neurons following retrograde transfection with RV-ChR2. $A$, Fluorescent corticospinal neurons in layer $5 B$ of mouse motor cortex, in a brain slice prepared several days after injection of RV-ChR2 in to the spinal cord. $\boldsymbol{B}$, Top, Example of a light-evoked spike in a transfected corticospinal neuron. Bottom, The average probability of evoking a spike ( $\left.p_{\text {spike }}\right)$ was 1 for transfected neurons (ChR2,$+ n=20$ ) and 0 for untransfected neurons $(C h R 2-, n=20) . C, p_{\text {spike }}$ as a function of stimulus intensity. Transfected corticospinal neurons $(n=9)$ were recorded while the intensity of the brief $(3 \mathrm{~ms})$ square pulse of light from a blue LED was varied by adjusting the amplitude of the command signal to the LED driver. $D$, $p_{\text {spike }}$ as a function of stimulus duration. Transfected neurons ( $n=20$ corticospinal neurons) were recorded while a square-pulse LED stimulus (power, $1.5 \mathrm{~mW} / \mathrm{mm}^{2}$ ) of variable duration $(1,3,5,10,20 \mathrm{~ms})$ was presented. The mean ( \pm SEM) for each stimulus value are plotted. $\boldsymbol{E}$, Cumulative distribution of the resting membrane potentials (RMPs) of transfected (ChR2+; $n=27)$ and untransfected (ChR2 $-; n=208)$ corticospinal neurons; differences were not statistically significant ( $p=0.43$, Kolmogorov-Smirnov test). $\boldsymbol{F}$, Cumulative distribution of the input resistances $\left(R_{\text {in }}\right)$ of transfected (ChR2 $\left.+; n=29\right)$ and untransfected (ChR2 $\left.-; n=226\right)$ corticospinal neurons; differences were not statistically significant $(p=0.86$, KolmogorovSmirnov test).

detect photocurrents in postsynaptic recordings from Venusnegative, bead-positive neurons.

This triple-labeling strategy thus enabled us to photostimulate the transfected subpopulation of corticospinal neurons while recording postsynaptically from untransfected corticospinal and corticostriatal neurons (crossed corticostriatal neurons, projecting across the corpus callosum to the contralateral striatum) in layer $5 \mathrm{~B}$, where these neurons are intermingled (Fig. $2 C, D$ ). This paradigm showed highly asymmetric connectivity: corticospinal neurons consistently projected to other corticospinal neurons (eight of eight experiments), but only weakly (one of eight experiments) to corticostriatal neurons (Fig. 2E,F). Overall, corticospinal connections to corticostriatal neurons were $8 \%$ as strong as to corticospinal neurons ( $p=0.0078$, signed rank test) (Fig. $2 F)$.

\section{$\mathrm{RV}$-ChR2 analysis of corticostriatal connections to corticospinal and corticostriatal neurons in layer 5B}

Next, we performed the complementary experiment: we injected $\mathrm{RV}$-ChR2 into the contralateral striatum to label corticostriatal neurons and render them photoexcitable (Fig. 3A), and again coinjected fluorescent tracers into the spinal cord and striatum to enable identification of untransfected corticospinal and corticostriatal neurons, respectively (Fig. $3 B, C$ ). In this case, because corticostriatal neurons in both hemispheres were labeled, the responses recorded in slices of motor cortex contralateral to the injection site came from both sources of crossed corticostriatal inputs- that is, from the ipsilateral axons of contralaterally la- beled corticostriatal neurons, and from the contralateral axons of ipsilaterally labeled corticostriatal neurons. Corticostriatal neurons consistently (eight of eight experiments) formed excitatory synaptic connections onto both corticospinal and corticostriatal neurons, in approximately equal proportion ( $p=0.95$, signed rank test) (Fig. 3E,F).

\section{RV-ChR2 analysis of connectivity with layer $5 \mathrm{~A}$ corticostriatal neurons}

In the preceding experiments, we recorded from corticospinal and corticostriatal neurons located side by side in layer 5B. However, corticostriatal neurons are also found in layer $5 \mathrm{~A}$, and layer $2 / 3$ pyramidal neurons have been observed to connect differentially to layer $5 \mathrm{~A}$ and $5 \mathrm{~B}$ corticostriatal neurons (Anderson et al., 2010), raising the possibility that these two types of corticostriatal neurons also have different connectivity with corticospinal neurons. To evaluate this, we RV labeled either corticospinal or corticostriatal neurons as before, and recorded from bead-labeled corticostriatal neurons in layer $5 \mathrm{~A}$, comparing them pairwise with corticospinal neurons in layer 5B in the same slices (Fig. 4). Again, corticospinal neurons connected strongly to corticospinal neurons, but weakly to layer $5 \mathrm{~A}$ corticostriatal neurons: input to layer $5 \mathrm{~A}$ corticostriatal neurons was on average $30 \%$ compared with corticospinal neurons ( $p=0.026$, signed rank test, $n=7$ ) (Fig. $4 A, B$ ). Similarly, corticostriatal neurons again connected approximately equally to corticospinal and layer $5 \mathrm{~A}$ corticostriatal neurons ( $p=0.84$, signed rank test, $n=6$ ) (Fig. $4 C, D)$. Thus, a similar pattern of asymmetric connectivity was also found for corticostriatal neurons in layer $5 \mathrm{~A}$.

\section{RV-ChR2 assay of corticospinal-corticortical connectivity}

The striking paucity of corticospinal-to-corticostriatal connections could be unique to this particular combination of neurons in motor cortex, or it could pertain to other classes of projection neurons. To explore this, we considered that callosally projecting corticocortical and corticostriatal neurons are closely related (Fame et al., 2011). Therefore, we injected RV-ChR2 into the spinal cord to transfect corticospinal neurons, and injected fluorescent beads into the contralateral spinal cord to label corticospinal neurons, and into the contralateral motor cortex to label callosally projecting corticocortical neurons (Fig. 5A). This paradigm showed highly asymmetric connectivity: corticospinal neurons consistently (five of five experiments) projected to other corticospinal neurons, but only weakly to corticocortical neurons (Fig. 5B,C). Overall, corticospinal-to-corticospinal connections were approximately sevenfold stronger than corticospinal-tocorticocortical connections ( $p=0.013$, paired $t$ test) (Fig. 5C). These experiments indicate that the asymmetric connectivity observed for corticostriatal-to-corticospinal connections applies to another subclass of callosal projection neurons.

We performed the complementary experiment, injecting RV$\mathrm{ChR} 2$ in the contralateral motor cortex to label presynaptic corticocortical neurons and axons; again, fluorescent beads were injected both into the contralateral spinal cord to label corticospinal neurons and into the contralateral motor cortex to label callosally projecting corticocortical neurons (Fig. $5 D$ ). The postsynaptic corticocortical neurons were located in multiple layers (layers $2 / 3,5 \mathrm{~A}$, and $5 \mathrm{~B}$ ). On average, corticocortical neurons formed excitatory synaptic connections onto both corticospinal and corticocortical neurons in approximately equal proportion ( $n=8$ experiments, $p=0.16$, paired $t$ test) (Fig. $5 E, F)$. Thus, overall these corticocortical-corticospinal experiments indicate 
A

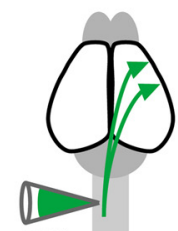

SPI
B

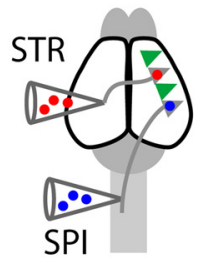

C

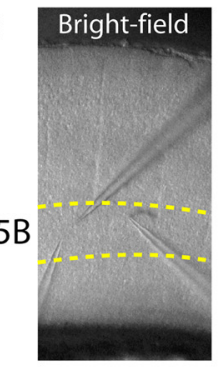

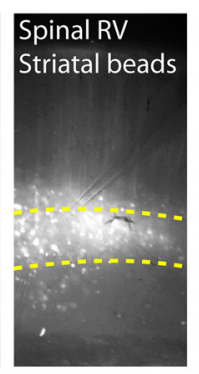

E

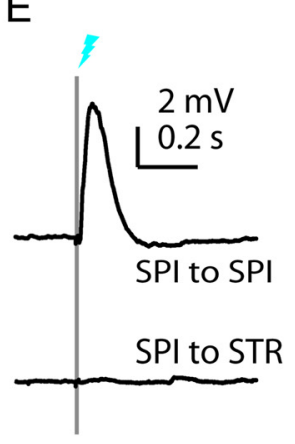

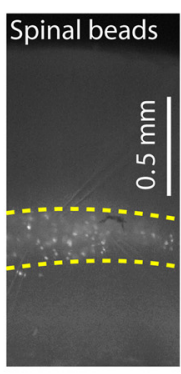

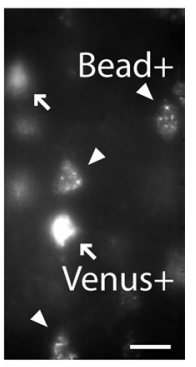

F

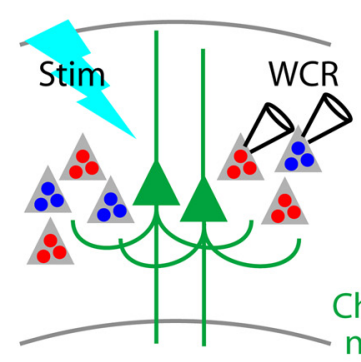

Postsynaptic: untransfected, bead-labeled:

STR

SPI

Presynaptic: ChR2+ corticospinal neurons and axons

Figure 2. Asymmetric output from photostimulated corticospinal neurons to untransfected corticospinal and corticostriatal neurons. A, Schematic depicting retrograde transfection of corticospinal neurons (SPI) with RV-ChR2. B, Schematic depicting retrograde anatomical labeling of corticospinal and corticostriatal (STR) neurons with fluorescent tracers. C, Images of brain slices of motor cortex containing the labeled neurons. Left to right: low-power bright-field, green (Venus and green beads), and red (red beads) fluorescence images. Green beads and red beads were injected into the striatum and spinal cord, respectively. Far right, Higher-magnification image showing readily distinguishable labeling patterns of transfected corticospinal neurons expressing Venus (arrows, (hR2 +) and other corticospinal neurons retrogradely labeled with fluorescent beads only (arrowheads). Scale bar, $25 \mu \mathrm{m}$. D, Schematic depicting the in vitro stimulation (Stim) and recording paradigm (WCR, whole cell recording). $\boldsymbol{E}$, Example responses to corticospinal stimulation, recorded simultaneously in untransfected corticospinal (blue) and corticostriatal (red) neurons. $\boldsymbol{F}$, Group analysis. Lines connect data points from individual pairs. Group means ( \pm SEM) are plotted to either side.

A

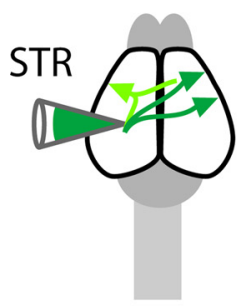

B

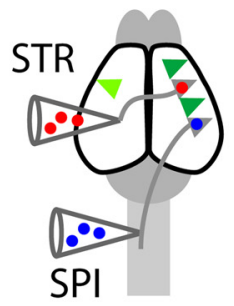

C

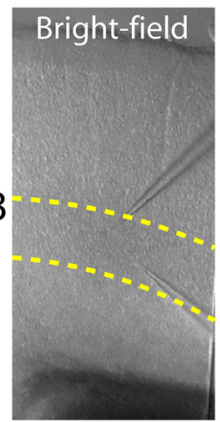

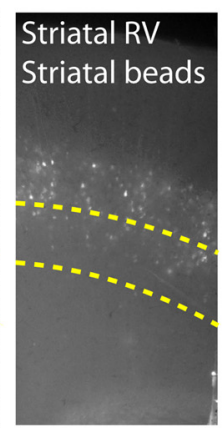

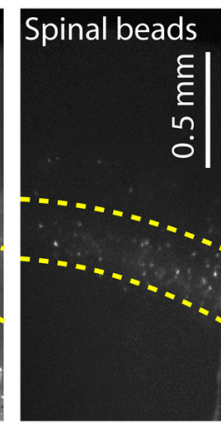

F Presynaptic:

$\mathrm{E}$

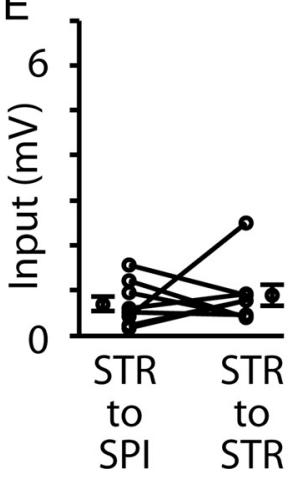

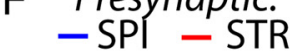

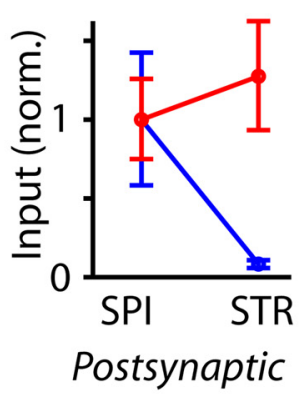

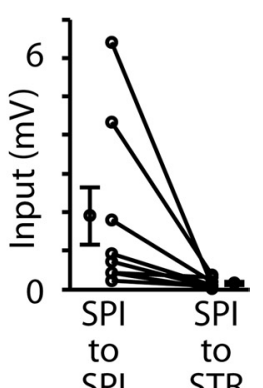

D

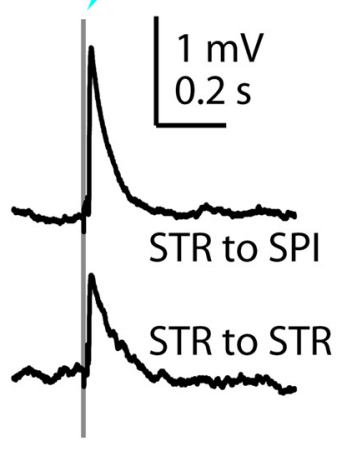

Figure 3. Shared output from photostimulated corticostriatal neurons to untransfected corticospinal and corticostriatal neurons. $\boldsymbol{A}$, Schematic depicting retrograde transfection of corticostriatal (STR) neurons with RV-ChR2. Corticostriatal neurons both contralateral (green) and ipsilateral (lighter shade of green) to the injection are retrogradely transfected; the latter can project across the callosum to the contralateral cortex and striatum. B, Schematic depicting retrograde anatomical labeling of corticospinal (SPI) and corticostriatal neurons with fluorescent tracers. $\boldsymbol{C}$, Images of brain slices of motor cortex containing the labeled neurons. Left to right: bright-field image, green fluorescent (Venus and green beads) image, and red fluorescent (red beads) image. Green beads and red beads were injected into the striatum and spinal cord, respectively. $\boldsymbol{D}$, Example responses to corticospinal stimulation, recorded simultaneously in untransfected corticospinal (blue) and corticostriatal (red) neurons. $\boldsymbol{E}$, Group analysis. Lines connect data points from individual pairs. Group means ( \pm SEM) are plotted to either side. $\boldsymbol{F}$, Group data for transfected presynaptic corticospinal neurons (from Fig. $2 F$ ) and corticostriatal neurons (from $\boldsymbol{E}$ ) are shown normalized to the value of the postsynaptic corticospinal response. 

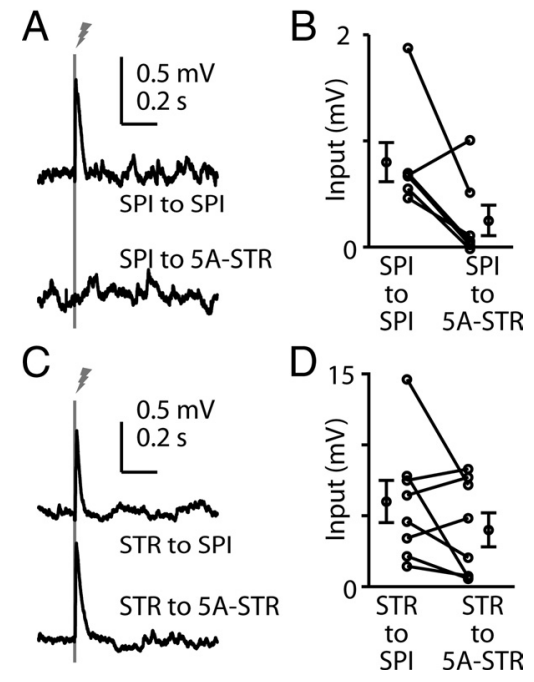

Figure 4. Asymmetric output from corticospinal to layer 5A corticostriatal neurons. $\boldsymbol{A}$, Example responses to corticospinal stimulation, recorded in a pair of untransfected corticospinal (SPI, blue) and layer 5A corticostriatal (5A-STR, red) neurons. B, Group analysis. Lines connect data points from individual pairs. Group means ( \pm SEM) are plotted to either side. $C$, Example responses to corticostriatal stimulation, recorded in a pair of untransfected corticospinal (blue) and layer 5 A corticostriatal (red) neurons. $\boldsymbol{D}$, Group analysis. Lines connect data points from individual pairs. Group means ( \pm SEM) are plotted to either side.

a pattern of asymmetric connectivity similar to that observed for corticostriatal-corticospinal connections.

\section{Paired recording analysis of the four potential pathways}

Finally, we used paired recordings to assess these results at the level of unitary connections. Simultaneous whole-cell recordings were made from various combinations of two, three, or four corticospinal and/or corticostriatal neurons (Fig. 6A,B). Connections were tested by driving presynaptic APs in one cell while monitoring responses in the other neurons (Fig. 6B, $C$ ).

Responses were analyzed to determine the connection probabilities $\left(p_{\text {con }}\right)$ for each type of connection. This analysis showed that corticospinal neurons connected to other corticospinal neurons (14/355), but did not make any connections to corticostriatal neurons (0/109); corticostriatal neurons, in contrast, made connections onto both corticospinal (24/115) and corticostriatal neurons (16/144) (Fig. 6D; Tables 1, 2).

Traces with detected EPSPs were analyzed to determine the amplitudes of unitary connections $\left(i_{\text {con }}\right)$. On average, $i_{\text {con }}$ did not differ by connection type for the three pathways $(p=0.49$, Kruskal-Wallis test) (Fig. 6E). We were also interested in estimating the overall relative pathway strength $\left(I_{\text {con }}\right)$ for each connection, both because (1) $I_{\text {con }}$ may be a more relevant measure of the aggregate connectivity between two populations of neurons, as it takes into account both the unitary connection probability and amplitude (i.e., $I_{\text {con }} \approx p_{\text {con }} i_{\text {con }}$ ); and (2) the responses recorded in the RV-ChR2 experiments represent $I_{\text {con }}$ (i.e., a combination of $p_{\text {con }}$ and $\left.i_{\text {con }}\right)$. We estimated $I_{\text {con }}$ by calculating the average response amplitude (in a short time window immediately following the first presynaptic action potential), for all traces. As expected, based on the lack of differences in $i_{\text {con }}$ (Fig. $6 D$ ), the profile of average $I_{\text {con }}$ values across the three connections (Fig. $6 F$ ) closely resembled that of the average $p_{\text {con }}$ values (Fig. 6D). These findings are thus consistent with the RV-ChR2 estimates of the relative connectivity strengths, but also extend those findings by indicating that corticospinal-to-corticospinal rates were lower compared with both corticostriatal-to-corticospinal and corticostriatal-to-corticostriatal connections (Tables 1, 2).

Waveforms of the different connections were further analyzed to extract information about the dynamic properties of EPSPs in these connections. This analysis was done on traces with $\mathrm{s} / \mathrm{n}$ ratios $>8$ (corticospinal-to-corticospinal, $n=11$; corticostriatalto-corticospinal, $n=23$; corticostriatal-to-corticostriatal, $n=$ 14). Example traces for connections in the three pathways for which unitary connections were detected (Fig. 7A) suggested several connection-specific differences. The decay time constants of the first EPSP were obtained by fitting an exponential function to the falling phase of the responses (15-50 ms poststimulus). The time constants were similar for corticospinal-to-corticospinal and corticostriatal-to-corticospinal connections, but were greater for corticostriatal-to-corticostriatal connections (Tukey post hoc comparisons, confidence level $\alpha=0.95$ with one-way ANOVA, $F_{(2,45)}=6.6$ and $p=0.0031$ ) (Fig. $7 B$ ). This difference is consistent with the observation that corticospinal neurons express high levels of hyperpolarization-activated current $\left(I_{h}\right)$, which filters synaptic inputs in these neurons (Sheets et al., 2011). Rise times of EPSPs (20$80 \%)$ did not differ significantly by connection type $(p=0.72$, $F_{(2,45)}=0.33$, one-way ANOVA).

Next, we evaluated whether the amplitudes of EPSPs changed over the course of short trains of responses in a connection-specific manner. Peak amplitudes of EPSPs of corticospinal-to-corticospinal connections increased, whereas those of corticostriatal-to-corticospinal connections decreased; those of corticostriatal-to-corticostriatal connections were intermediate (Fig. 7C,D). In other words, corticospinal responses were facilitating for corticospinal inputs but depressing for corticostriatal inputs. Peak EPSP amplitude, however, can reflect synaptic facilitation/depression, temporal summation, or both processes. Therefore, we also determined the trough values in the trains of EPSPs (Fig. 7C). Trough values were highest for corticostriatal-to-corticostriatal connections (Fig. 7E), suggesting more effective temporal summation associated with postsynaptic corticostriatal neurons. Subtraction of these trough values from the peak amplitudes (Fig. $7 F$ ) showed that greater values for corticospinal-to-corticospinal responses compared with corticostriatal-to-corticospinal or corticostriatal-to-corticostriatal connections, indicating that connections were more depressing for presynaptic corticostriatal neurons, compared with presynaptic corticospinal neurons.

\section{Discussion}

By transfecting projection neurons with ChR2, we were able to stimulate either corticospinal or corticostriatal neurons, and by recording from identified untransfected corticospinal and corticostriatal neurons in the same slices, we were able to determine the relative strengths of connections in the excitatory microcircuit formed by the four potential synaptic pathways within and across these two cell classes. Corticospinal and corticostriatal neurons both formed recurrent (within-class) connections, but across-class connectivity was overwhelmingly corticostriatal-tocorticospinal in direction. Paired recordings corroborated these connectivity patterns and revealed additional functional specializations in these pathways.

\section{$\mathrm{RV}-\mathrm{ChR} 2$ as a tool for functional microcircuit analysis}

$\mathrm{RV}$-ChR2 was an efficient way to transfect cortical projection neurons, making them photoexcitable. Combined with wholecell recordings, this technique provided single-cell resolution on the postsynaptic end of the connections being tested, but differed 

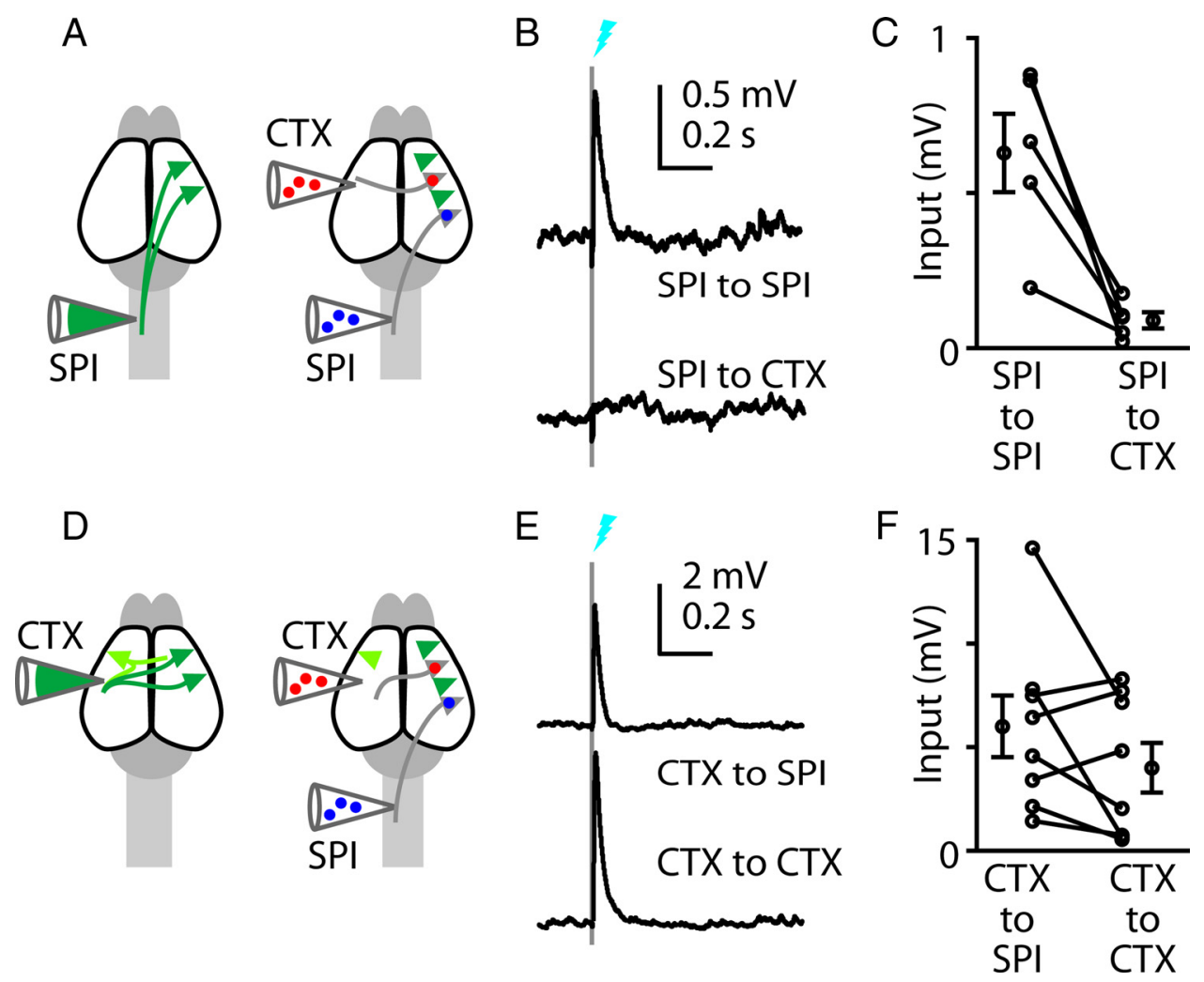

Figure 5. Asymmetric output from photostimulated corticospinal neurons to untransfected corticospinal and corticocortical neurons. A, Schematics depicting retrograde transfection of corticospinal (SPI) neurons with RV-ChR2 (left), and retrograde anatomical labeling of corticospinal and corticocortical (CTX) neurons with fluorescent tracers (right). B, Example responses to corticospinal stimulation, recorded simultaneously in untransfected corticospinal (blue) and corticocortical (red) neurons. C, Group analysis. Lines connect data points from individual pairs. Group means ( \pm SEM) are plotted to either side. $\boldsymbol{D}$, Schematics depicting retrograde transfection of corticocortical neurons with RV-ChR2 (left), and retrograde anatomical labeling of corticospinal and corticocortical neurons with fluorescent tracers (right). $\boldsymbol{E}$, Example responses to callosal corticocortical stimulation, recorded simultaneously in untransfected corticospinal (blue) and corticocortical (red) neurons. $\boldsymbol{F}$, Group analysis. Lines connect data points from individual pairs. Group means ( \pm SEM) are plotted to either side.

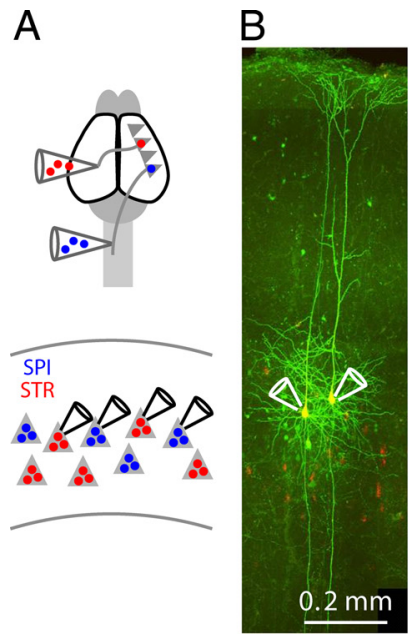

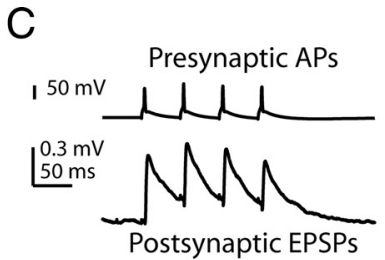

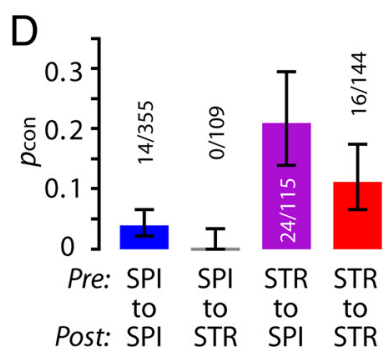

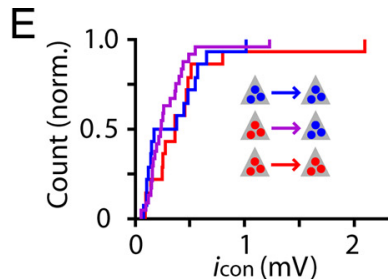

$\mathrm{F}$

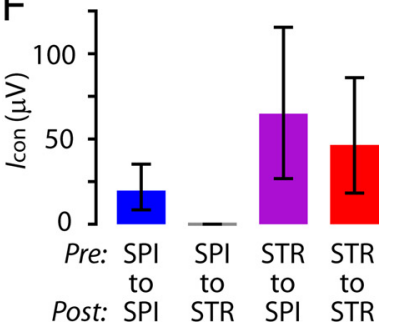

Figure 6. Paired recordings also show asymmetric connectivity in the corticospinal-corticostriatal microcircuit. $A$, Schematic showing retrograde anatomical labeling and recording paradigm. SPI, corticospinal; STR, corticostriatal. $\boldsymbol{B}$, Example image of two corticospinal neurons filled with fluorescent dye during paired recording. $\boldsymbol{C}$, Example of a unitary synaptic connection between two corticospinal neurons. $D, p_{\text {con }}$ values. Error bars indicate binomial $95 \%$ Cl. $E$, Cumulative histogram of unitary EPSP amplitude $\left(i_{\text {con }}\right)$, for the first EPSP in the train. $\boldsymbol{F}, I_{\text {con }}$.

from paired recordings in that the aggregate output from many presynaptic inputs could be sampled at once. Another difference relates to the severing of axons in cortical slices: paired recordings inevitably underestimate connectivity because intact axons are required to detect connections, but this requirement does not pertain to the optogenetic assay due to the photoexcitability of ChR2-expressing axons. RV-ChR2 yields information about relative connectivity, and for quantification it was necessary to compare responses in different postsynaptic neurons. Thus, this technique is useful in tandem with paired recordings, which are more laborious but provide additional information about unitary connectivity including dynamic properties.

RV-ChR2 enabled selective activation of a specific subpopulation of neurons defined by their axonal projection target. This 
Table 1. Statistical analysis of paired recording data: connection probabilities

\begin{tabular}{lll}
\hline & Presynaptic & \\
\cline { 2 - 3 } Postsynaptic & Corticospinal & Corticostriatal \\
\hline Corticospinal & Pathway: SPI $\rightarrow$ SPI & Pathway: STR $\rightarrow$ SPI \\
& $n_{\text {connected }: 14}:$ connected: $: 24$ \\
& $n_{\text {tested }}: 355$ & $n_{\text {tested }}: 115$ \\
& $p_{\text {con }}: 0.04$ & $p_{\text {con }}: 0.21$ \\
Corticostriatal & Pathway: SPI $\rightarrow$ STR & Pathway: STR $\rightarrow$ STR \\
& $n_{\text {connected }}: 0$ & $n_{\text {connected }}: 16$ \\
& $n_{\text {tested }}: 109$ & $n_{\text {tested }}: 144$ \\
& $p_{\text {con }}: 0$ & $p_{\text {con }}: 0.11$ \\
\hline
\end{tabular}

Connection probabilities ( $\left.p_{\text {con }}\right)$ were calculated as the number of connections detected $\left(n_{\text {connected }}\right)$ divided by the total number of connections tested ( $\left.n_{\text {tested }}\right)$. SPI, corticospinal; STR, corticostriatal.

Table 2. Statistical analysis of paired recording data: comparison of connectivity

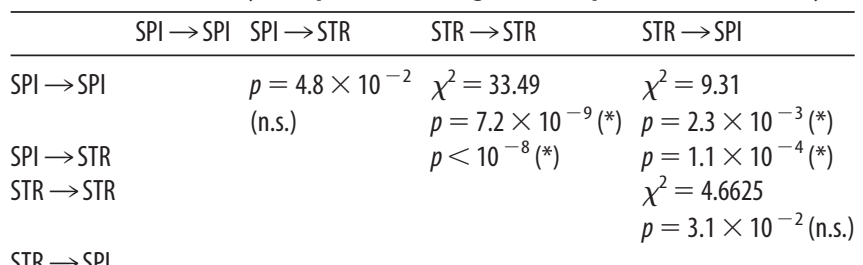

$\mathrm{STR} \rightarrow \mathrm{SPI}$

Pearson's $\chi^{2}$ test and, when an entry in contingency table was $<10$, Fisher's exact test were used to test the independence of $p_{\text {con }}$ on presynaptic and postsynaptic cell types. The $\chi^{2}$ statistic and $p$ values were calculated, and the Bonferroni method ( $p<0.05 / 6$ was considered significant) was used for multiple comparisons. SPI, corticospinal; STR, corticostriatal.

retrograde aspect makes RV-ChR2 particularly useful for analysis of neocortical pyramidal neurons, whose functional roles are directly related to their long-range connectivity. In this respect, $\mathrm{RV}$-ChR2 contrasts with other transfection and labeling methods such as anterograde viral labeling and transgenic models, which label neurons based on other anatomical or genetic attributes.

One potential issue involved in retrograde labeling with RVChR2 is that the transfected neurons express ChR2 in their axons; these axons, including severed axons, can therefore contribute to the observed responses. This is a consideration in experimental design because cortical neurons can project to multiple longrange targets. Here, for example, injection into the striatum in one hemisphere resulted in transfection of corticostriatal neurons in both hemispheres. Consequently, photostimulation of the contralateral motor cortex resulted in activation of both ipsilaterally and contralaterally located crossed (callosally projecting) corticostriatal neurons: that is, (1) cell bodies, dendrites, and axons of neurons whose cell bodies were located in the slice (i.e., contralateral to the injection); and (2) axons of neurons whose cell bodies were located in the injected hemisphere. Despite this mixture, the RV-ChR2 experiments and paired-recording experiments gave similar results, suggesting that the connectivity of corticostriatal axons was similar for their local and contralateral projections. This is furthermore consistent with previous observations of similar connectivity for the ipsilateral and contralateral axonal projections of corticocortical neurons (Petreanu et al., 2007; Anderson et al., 2010).

\section{Four-way specialization in the corticospinal-corticostriatal microcircuit \\ Corticospinal-to-corticospinal}

These connections were present but less frequent compared with corticostriatal-to-corticostriatal recurrent connections. The functional properties were distinct: individual EPSPs were fast decaying, and trains of EPSPs showed relatively less synaptic depression. The faster decay appears to be a corticospinal-specific postsynaptic property, because it was also observed for corticostriatal-to-corticospinal connections, and likely reflects corticospinal-specific $I_{\mathrm{h}}$ (Sheets et al., 2011). The relatively greater facilitation appears to be a corticospinal-specific presynaptic property, because it was not observed in the other two connection types, which involved presynaptic corticostriatal neurons. This facilitation is consistent with observations based on paired recordings from thick-tufted layer 5B pyramidal neurons in rat somatosensory cortex (Williams and Atkinson, 2007).

Corticospinal recurrent connections are of particular interest from the standpoint of cortical mechanisms of motor control (Phillips, 1959; Phillips and Porter, 1977; Matsumura et al., 1996; Smith and Fetz, 2009). The relatively low connectivity rates observed here for these connections are consistent with the idea that corticospinal neurons connect in a limited and presumably selective manner with one another (Schieber, 2001).

Spinal axons of corticospinal neurons make facilitating synapses onto spinal motoneurons (Phillips and Porter, 1964). Consistent with this, we found relatively facilitating connections among corticospinal neurons, an effect that was, however, attenuated by postsynaptic $I_{\mathrm{h}}$. Facilitation could boost activity in subnetworks of interconnected neurons during sustained activity (Wang et al., 2006). The corticospinal/corticostriatal differences in short-term plasticity observed here parallel differences in firing patterns: in mouse motor cortex, corticospinal neurons tend to fire tonically, at sustained or even accelerating rates, while corticostriatal neurons tend to fire phasically, showing spike frequency adaptation (B. A. Suter and G. M. G. Shepherd, unpublished observations), differences also previously reported for projection neurons in other cortical areas and species (Hattox and Nelson, 2007; Miller et al., 2008; Otsuka and Kawaguchi, 2008; Brown and Hestrin, 2009; Dembrow et al., 2010). The effects of sustained activity on corticospinal and corticostriatal neurons could be magnified by the combined effects of the shortterm synaptic properties and firing properties.

\section{Corticospinal-to-corticostriatal}

This connection was largely absent, precluding analysis of dynamic properties. A similar lack of connections was found on testing the potential pathway from corticospinal neurons to another class of callosal projection neurons, corticocortical neurons. The "rectifying" connectivity between corticostriatal and corticospinal neurons implies that corticospinal neurons are compartmentalized at a downstream position within the excitatory intracortical network.

\section{Corticostriatal-to-corticostriatal}

Of significance because they also represent recurrent excitation, these connections were characterized by (1) longer EPSP decay kinetics, associated with greater temporal dendritic summation, a corticostriatal-specific postsynaptic property; and (2) greater depression during trains of EPSPs, a corticostriatal-associated presynaptic property. Recurrent connections among corticostriatal neurons have been found in other species and cortical areas (Morishima and Kawaguchi, 2006; Brown and Hestrin, 2009; Morishima et al., 2011), and our data are consistent with the relatively high connection rates reported. The synaptic depression we observed for these connections is furthermore consistent with that observed for connections between another type of intracerebrally projecting neurons (ICPNs), layer $2 / 3$ neurons in somatosensory cortex (Williams and Atkinson, 2007). 

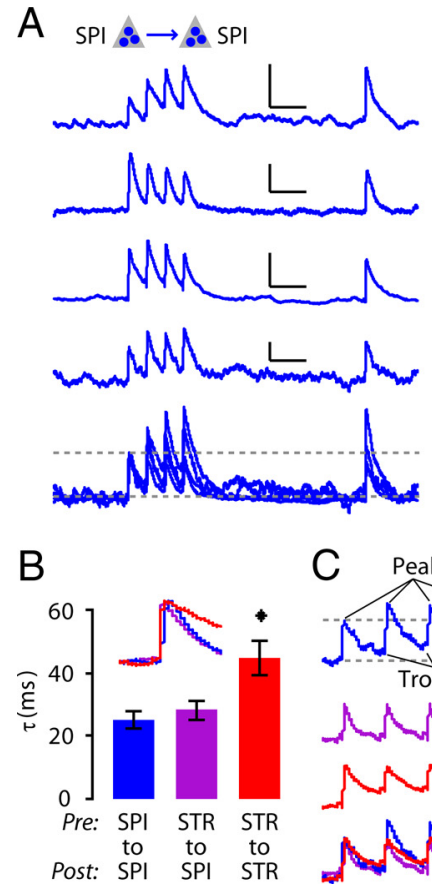
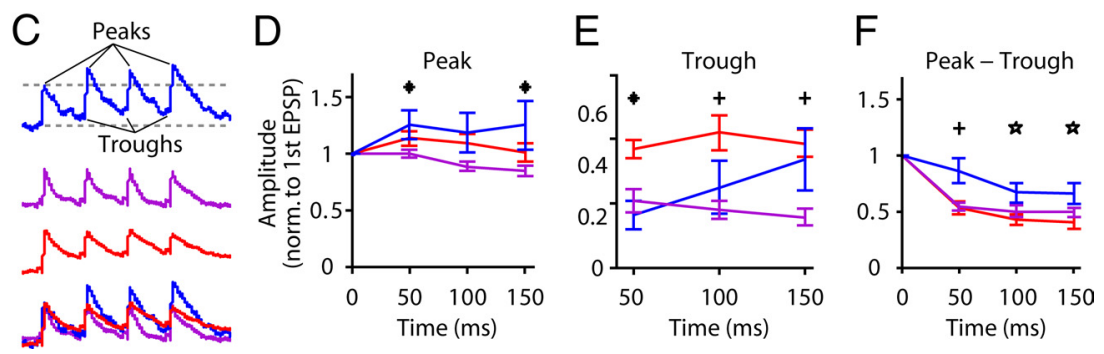

Figure 7. Dynamic properties of unitary EPSPS. $\boldsymbol{A}$, Examples of unitary connections for corticospinal-to-corticospinal (left), corticostriatal-to-corticostriatal (middle), and corticostriatal-tocorticostriatal (right). SPI, corticospinal; STR, corticostriatal. Examples are plotted individually (top four traces), and together (bottom) with normalization to the first EPSP peak and baseline (upper and lower dashed lines). Horizontal scale bar, $100 \mathrm{~ms}$. B, EPSP decay time constant $(\tau)$, for the first EPSP. C, Examples of responses indicating EPSP peaks and troughs (minimum voltage before next EPSP). Bottom, Overlay of traces, normalized to first EPSP. D, Peak amplitudes of the EPSPs in the train, normalized to the amplitude of the first EPSP. Asterisks $\left.{ }^{*}\right)$ indicate significant differences between corticospinal-to-corticospinal and corticostriatal-to-corticospinal connections (Tukey's post hoc comparisons, confidence level $\alpha=0.95$ with one-way ANOVA). $E$, Trough amplitudes, normalized to the amplitude of the first EPSP. Asterisk $\left(^{*}\right.$ ) indicates significant differences between corticospinal-to-corticospinal and both other connections (corticostriatal-to-corticospinal and corticostriatal-to-corticostriatal), and crosses ( + ) indicate significant differences between corticostriatal-to-corticostriatal and corticostriatal-to-corticospinal connections (Tukey post hoc comparisons, confidence level $\alpha=0.95$ with one-way ANOVA). $\boldsymbol{F}$, Trough-subtracted peak EPSP amplitudes, normalized to the amplitude of the first EPSP. (ross $(+)$ indicates significant differences between corticospinal-to-corticospinal and both other connections (corticostriatal-to-corticospinal and corticostriatal-to-corticostriatal), and asterisks (*) indicate significant differences between corticospinal-to-corticospinal and corticostriatal-to-corticostriatal connections (Tukey's post hoc comparisons, confidence level $\alpha=0.95$ with one-way ANOVA).

Higher order Lower order

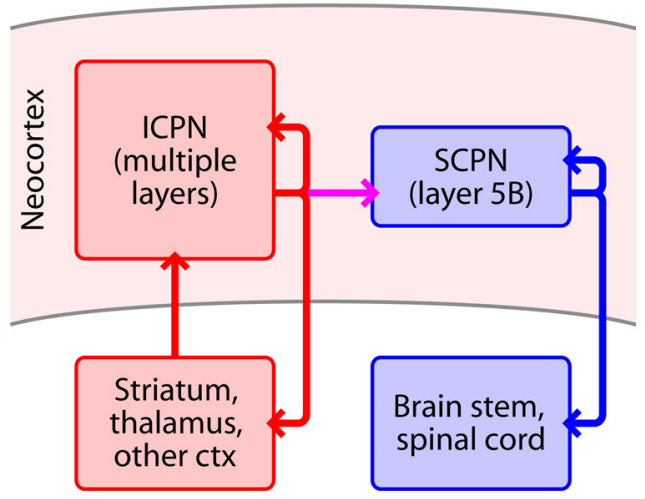

Figure 8. Hierarchical organization of the excitatory microcircuit formed by corticospinal and corticostriatal neurons. ICPNs include (callosally projecting) corticostriatal and corticocortical neurons. SCPNs include corticospinal neurons. Excitation flows unidirectionally from corticostriatal to corticospinal neurons.

\section{Corticostriatal-to-corticospinal}

These connections occurred at a rate similar to recurrent corticostriatal connections, and their dynamic behavior reflected the combination of the corticostriatal-associated presynaptic property of more depressing short-term plasticity in EPSP trains with the corticospinal-associated postsynaptic property of more attenuating EPSP kinetics, resulting in an overall maximally phasic (as opposed to tonic) pattern of responses. The synaptic depres- sion we observed in these connections is again consistent with that observed for connections from layer $2 / 3$ neurons to thicktufted layer $5 \mathrm{~B}$ neurons in somatosensory cortex (Williams and Atkinson, 2007). From a connectional standpoint, this connection was significant because these were the only major form of across-class connections observed for these two cell types. An implication is that during sustained activity, communication could decrease relatively faster over this across-class channel than for the two recurrent pathways. Whether this channel represents "feedforward" excitation is unclear, but from a systems perspective it may be relevant that corticostriatal activity is associated more with the planning/preparatory stages of movement and corticospinal activity more with the execution stages (Turner and DeLong, 2000; Monchi et al., 2006; Lemon, 2008; Shadmehr and Krakauer, 2008).

\section{Comparison to previous studies suggests a generic} microcircuit formed by intracerebrally and subcerebrally projecting pyramidal neurons

Extremely asymmetric connectivity between projection neurons was reported in a study in rat prefrontal cortex (Morishima and Kawaguchi, 2006; Morishima et al., 2011), with almost no corticopontine-to-corticostriatal connections detected (1 of 96 connections tested). Asymmetric across-class connectivity has, however, also been shown for projection neurons in mouse visual cortex: corticotectal-to-corticocortical connections about four times less common than corticocortical-to-corticotectal connections (Brown and Hestrin, 2009). Considered in the context of these previous observations, our findings illuminate a clear 
pattern: the directionality is consistently from ICPNs (i.e., intratelencephalic type, callosally projecting corticostriatal and/or corticocortical neurons) onto subcerebrally projecting neurons (SCPNs) (i.e., pyramidal tract type neurons, e.g., corticospinal, corticopontine, or corticotectal) (Molyneaux et al., 2007; Reiner et al., 2010) (Fig. 8). Interestingly, although the long-range targets of SCPNs vary widely in the adult, they initially project an axon to the spinal cord (Stanfield et al., 1982; Stanfield and O'Leary, 1985; Heffner et al., 1990). Their common developmental origin strengthens the case for unidirectional across-class connectivity in a generic ICPN-SCPN microcircuit.

From a motor systems viewpoint, we note that hierarchical organization is a prominent aspect of subcortical motor circuits (Shepherd, 1994; Swanson, 2000); our findings extend this concept into the cortex, by showing that intracortical circuits in motor cortex also are hierarchically organized, with ICPNs upstream of SCPNs (Fig. 8). In the case of corticospinal neurons, we further speculate that the downstream compartmentalization of these SCPNs in combination with corticospinal-specific $I_{\mathrm{h}}$ neuromodulation (Sheets et al., 2011) presents a way in which ongoing intracortical activity in motor cortex may be flexibly associated with signaling to the spinal cord; this arrangement potentially represents a mechanism for "dissociating motor cortex from the motor" (Schieber, 2011).

Within-class interconnections have been evaluated for many subtypes of both the ICPN and SCPN superclasses. For the ICPN superclass, these include callosally projecting corticostriatal neurons in rat prefrontal cortex (Morishima and Kawaguchi, 2006; Morishima et al., 2011) and mouse somatosensory cortex (Brown and Hestrin, 2009), and corticocortical neurons in rat somatosensory cortex (Le Bé et al., 2007) and mouse visual cortex (Brown and Hestrin, 2009). For the SCPN superclass, these include corticopontine neurons in rat prefrontal cortex (Morishima and Kawaguchi, 2006; Morishima et al., 2011) and corticotectal neurons in mouse visual cortex (Brown and Hestrin, 2009). Within-class connections have been consistently observed, indicating that these recurrent excitatory synaptic pathways are also consistent elements in ICPN-SCPN microcircuits (Fig. 8).

The dynamic properties of unitary connections formed between various classes of projection neurons have also been examined in a recent analysis of connections in rat prefrontal cortex (Morishima et al., 2011), demonstrating presynaptic-specific properties (e.g., short-term facilitation associated with presynaptic corticostriatal neurons), but not postsynaptic-specific properties such as the faster-decaying EPSPs we recorded in corticospinal neurons. Another study found weaker recurrent connections for retrogradely labeled corticocortical neurons compared with anatomically identified thick tufted pyramidal neurons (SCPN type) in juvenile rat sensory cortex (Le Bé et al., 2007). Analysis of connections in mouse sensory areas (Brown and Hestrin, 2009) showed no significant differences in pair/ pulse ratios or other synaptic properties. Thus, it appears that while the basic connectivity patterns may be conserved, the dynamic properties of the different types of connections in the SCPN-ICPN microcircuit may be modified in different cortical areas, projection subtypes, and species.

\section{References}

Amassian VE, Stewart M (2003) Motor cortical and other cortical interneuronal networks that generate very high frequency waves. Suppl Clin Neurophysiol 56:119-142.

Anderson CT, Sheets PL, Kiritani T, Shepherd GMG (2010) Sublayer- specific microcircuits of corticospinal and corticostriatal neurons in motor cortex. Nat Neurosci 13:739-744.

Brown SP, Hestrin S (2009) Intracortical circuits of pyramidal neurons reflect their long-range axonal targets. Nature 457:1133-1136.

Dembrow NC, Chitwood RA, Johnston D (2010) Projection-specific neuromodulation of medial prefrontal cortex neurons. J Neurosci 30:16922-16937.

Fame RM, MacDonald JL, Macklis JD (2011) Development, specification, and diversity of callosal projection neurons. Trends Neurosci 34:41-50.

Georgopoulos AP, Stefanis CN (2010) The motor cortical circuit. In: Handbook of brain microcircuits (Shepherd GM, Grillner S, eds), pp 39-45. New York: Oxford UP.

Hattox AM, Nelson SB (2007) Layer V neurons in mouse cortex projecting to different targets have distinct physiological properties. J Neurophysiol 98:3330-3340.

Heffner CD, Lumsden AG, O'Leary DD (1990) Target control of collateral extension and directional axon growth in the mammalian brain. Science 247:217-220

Kaneko T, Caria MA, Asanuma H (1994) Information processing within the motor cortex. I. Responses of morphologically identified motor cortical cells to stimulation of the somatosensory cortex. J Comp Neurol 345:161-171.

Kaneko T, Cho R, Li Y, Nomura S, Mizuno N (2000) Predominant information transfer from layer III pyramidal neurons to corticospinal neurons. J Comp Neurol 423:52-65.

Le Bé JV, Silberberg G, Wang Y, Markram H (2007) Morphological, electrophysiological, and synaptic properties of corticocallosal pyramidal cells in the neonatal rat neocortex. Cereb Cortex 17:2204-2213.

Lemon RN (2008) Descending pathways in motor control. Annu Rev Neurosci 31:195-218.

Lemon RN (2010) What drives corticospinal output? F1000 Biol Rep 2:51.

Matsumura M, Chen D, Sawaguchi T, Kubota K, Fetz EE (1996) Synaptic interactions between primate precentral cortex neurons revealed by spike-triggered averaging of intracellular membrane potentials in vivo. J Neurosci 16:7757-7767.

Miller MN, Okaty BW, Nelson SB (2008) Region-specific spike-frequency acceleration in layer 5 pyramidal neurons mediated by Kvl subunits. J Neurosci 28:13716-13726.

Molyneaux BJ, Arlotta P, Menezes JR, Macklis JD (2007) Neuronal subtype specification in the cerebral cortex. Nat Rev Neurosci 8:427-437.

Monchi O, Petrides M, Strafella AP, Worsley KJ, Doyon J (2006) Functional role of the basal ganglia in the planning and execution of actions. Ann Neurol 59:257-264.

Morishima M, Kawaguchi Y (2006) Recurrent connection patterns of corticostriatal pyramidal cells in frontal cortex. J Neurosci 26:4394-4405.

Morishima M, Morita K, Kubota Y, Kawaguchi Y (2011) Highly differentiated projection-specific cortical subnetworks. J Neurosci 31:1038010391.

Nagai T, Ibata K, Park ES, Kubota M, Mikoshiba K, Miyawaki A (2002) A variant of yellow fluorescent protein with fast and efficient maturation for cell-biological applications. Nat Biotechnol 20:87-90.

Osakada F, Mori T, Cetin AH, Marshel JH, Virgen B, Callaway EM (2011) New rabies virus variants for monitoring and manipulating activity and gene expression in defined neural circuits. Neuron 71:617-631.

Otsuka T, Kawaguchi Y (2008) Firing-pattern-dependent specificity of cortical excitatory feedforward subnetworks. J Neurosci 28:11186-11195.

Petreanu L, Huber D, Sobczyk A, Svoboda K (2007) Channelrhodopsin-2assisted circuit mapping of long-range callosal projections. Nat Neurosci 10:663-668.

Phillips CG (1959) Actions of antidromic pyramidal volleys on single Betz cells in the cat. Q J Exp Physiol Cogn Med Sci 44:1-25.

Phillips CG, Porter R (1964) The pyramidal projection to motoneurones of some muscle groups of the baboon's forelimb. Prog Brain Res 12:222-245.

Phillips CG, Porter R (1977) Corticospinal neurones: their role in movement. London: Academic.

Rathelot JA, Strick PL (2006) Muscle representation in the macaque motor cortex: an anatomical perspective. Proc Natl Acad Sci U S A 103:82578262.

Reiner A, Hart NM, Lei W, Deng Y (2010) Corticostriatal projection neurons- dichotomous types and dichotomous functions. Front Neuroanat 4:142. 
Schieber MH (2001) Constraints on somatotopic organization in the primary motor cortex. J Neurophysiol 86:2125-2143.

Schieber MH (2011) Dissociating motor cortex from the motor. J Physiol 589:5613-5624.

Shadmehr R, Krakauer JW (2008) A computational neuroanatomy for motor control. Exp Brain Res 185:359-381.

Sheets PL, Suter BA, Kiritani T, Chan CS, Surmeier DJ, Shepherd GMG (2011) Corticospinal-specific HCN expression in mouse motor cortex: Ih-dependent synaptic integration as a candidate microcircuit mechanism involved in motor control. J Neurophysiol 106:2216-2231.

Shepherd GM (1994) Motor hierarchies. In: Neurobiology, pp 452-477. New York: Oxford UP.

Smith WS, Fetz EE (2009) Synaptic interactions between forelimb-related motor cortex neurons in behaving primates. J Neurophysiol 102:1026-1039.

Stanfield BB, O'Leary DD (1985) The transient corticospinal projection from the occipital cortex during the postnatal development of the rat. J Comp Neurol 238:236-248.

Stanfield BB, O'Leary DD, Fricks C (1982) Selective collateral elimination in early postnatal development restricts cortical distribution of rat pyramidal tract neurones. Nature 298:371-373.

Suter BA, O'Connor T, Iyer V, Petreanu LT, Hooks BM, Kiritani T, Svoboda K, Shepherd GMG (2010) Ephus: multipurpose data acquisition software for neuroscience experiments. Front Neural Circuits 4:100.
Swanson LW (2000) Cerebral hemisphere regulation of motivated behavior. Brain Res 886:113-164.

Tseng GF, Parada I, Prince DA (1991) Double-labelling with rhodamine beads and biocytin: a technique for studying corticospinal and other projection neurons in vitro. J Neurosci Methods 37:121-131.

Turner RS, DeLong MR (2000) Corticostriatal activity in primary motor cortex of the macaque. J Neurosci 20:7096-7108.

Wang Y, Markram H, Goodman PH, Berger TK, Ma J, Goldman-Rakic PS (2006) Heterogeneity in the pyramidal network of the medial prefrontal cortex. Nat Neurosci 9:534-542.

Weiler N, Wood L, Yu J, Solla SA, Shepherd GMG (2008) Top-down laminar organization of the excitatory network in motor cortex. Nat Neurosci 11:360-366.

Wickersham IR, Finke S, Conzelmann KK, Callaway EM (2007) Retrograde neuronal tracing with a deletion-mutant rabies virus. Nat Methods 4:47-49.

Wickersham IR, Sullivan HA, Seung HS (2010) Production of glycoproteindeleted rabies viruses for monosynaptic tracing and high-level gene expression in neurons. Nat Protoc 5:595-606.

Williams SR, Atkinson SE (2007) Pathway-specific use-dependent dynamics of excitatory synaptic transmission in rat intracortical circuits. J Physiol 585:759-777. 